\begin{tabular}{|c|c|c|}
\hline II & $85^{\circ} 2$ & $28^{\prime} 45^{\prime \prime} 8$ \\
\hline$\tau$ & 310 & 041. \\
\hline$\beta$ & 55 & 8 \\
\hline & & 17 \\
\hline $\boldsymbol{P}$ & 74 & 4156 \\
\hline $\log$ & 0.380 & 06001 \\
\hline & $953^{\prime \prime} \mathrm{C}$ & 030 \\
\hline
\end{tabular}

Dr. L. Gruber.

Mittleres Aequinox 1874.0.

Epoche 1874, Juni 29.0 mittlere Berliner Zeit.

$\begin{array}{lrrrr}\mathrm{L} & 269^{\circ} & 51^{\prime} & 16^{\prime \prime} 0 \\ \mathrm{M} & 319 & 16 & 31.0 \\ \pi & 310 & 34 & 45.0 \\ \Omega & 54 & 56 & 15.0 \\ \mathrm{i} & 3 & 15 & 48.3 \\ \varphi & 8 & 29 & 29.9\end{array}$

log a 0.385548

$\mu \quad 936^{\prime \prime} 883$

Oberingenieur a. D. C. W. Plath. Mittleres Aequinox 1880.0.

Epoche 1875, November 11.5 mittlere Berliner /eit.

L $\quad 40^{\circ} 3^{\prime} 23^{\prime \prime} 49$

M $\quad 88 \quad 4027.05$

$\pi \quad 3112256.44$

$8 \quad 54 \quad 54 \quad 34.30$

i $\quad 31425.98$

$\phi \quad 9 \quad 6 \quad 5.00$

$\log$ a 0.3880548

$\mu \quad 928^{\prime \prime} 8046$

Hamburg, im August 1875.

C. W. Plath, Oberingenieur a. D.

\title{
Schreiben des Herrn Dr. C. W. Moesta an den Herausgeber.
}

Im Folgenden erlaube ich mir, Ihnen eine Vergleichung der in Santiago von $1853-1860$ beobachteten Sternpositionen mit denen des neuen Sterncatalogs von Washington (1873) zu übersenden. Dieser Catalog enthält bekanntlich alle von $1845-1871$ in Washington beobachteten Sterne, unter denen sich eine grosse Anzabl südlicher Sterne findet, deren Declinationen bis $-40^{\circ}$ und zum Theil darïber hinausreichen. Die Vergleichung bezieht sich nur auf die Declinationen, und da die mittlere Epoche für Washington wenig verschieden von der für Santiago ist, so konnten die constanten Differenzen beider Cataloge auch für Sterne südlich vom Zenith von Santiago, deren eigene Bewegungen nur unvollkommen oder gänzlich unbekannt sind, mit ziemlich grosser Sicherheit ermittelt werden. Zunächst wählte ich als Vergleichungspunkte die grösseren Sterne nördlich vom Zenith von Santiago, die vielfach beobachtet worden und deren eigene Bewegungen genau bekannt sind. Eine Zusammenstellung solcher Sterne, nach ihren Poldistanzen geordnet und mit verschiedenen Catalogen verglichen, findet sich auf pag. XXXVI der Einleitung zum 1. Bande meiner Beobachtungen. Von diesen Sternen habe ich bei der vorliegenden Vergleichung diejenigen ausgeschlossen, welche in geringerer Zahl beobachtet sind, und ausserdem die Poldistanz von $\beta$ Aquilae um 0"23 verkleinert, welche Verbesserung sich durch cine neue Reduction der Beobachtungen herausstellte. Indem ich nun die Washingtoner Beobachtungen mit den von Laugier gege-

benen eigenen Bewegungen auf 1855.0 reducirte, ergeben sich die im beistebenden Tableau aufgeführten Differenzen: Zahl d. Beob. Zahl d. Beob.

$\alpha$ Piscis aust.

$\alpha$ Scorpii

$\beta$ Corvi

$\mu^{1}$ Sagittarii

$\beta^{\prime}$ Scorpii

$\beta$ Ceti

$\alpha^{2}$ Librae

$\delta$ Hydrae

15 Argus

$\alpha^{2}$ Capricorni

$\alpha$ Virginis

$\beta$ Librae

$\beta$ Orionis

$\beta$ Aquarii

$\delta$ Ophiuchi

a Aquarii

$\delta$ Aquilae

$\alpha$ Ceti

$\boldsymbol{\beta}$ Aquilae

$\alpha$

$\alpha$ Leonis

द) Aquilae

$\beta$ Leonis

$\delta$ n
Santiago $P$

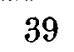

25

44

17

7

30

14

24

3

33

41

10

16

16

18

12

15

22

20

27

37

19

44

17

14

\section{oldistanz Wash. S. -W.} $120^{\circ} 23^{\prime} \quad 159 \quad-0^{\prime \prime} 83$ $\begin{array}{llll}116 & 6 & 84 & -0.66\end{array}$ $\begin{array}{llll}112 & 25 & 37 & -1.23\end{array}$ $\begin{array}{llll}111 & 5 & 18 & -1.22\end{array}$ $\begin{array}{llll}10924 & 61 & -1.17\end{array}$ $\begin{array}{lll}108 & 46 \quad 37 \quad t-0.13\end{array}$

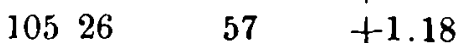
$10359 \quad 46 \quad-0.73$ $\begin{array}{llll}103 & 53 \quad 47 \quad-1.06\end{array}$ $\begin{array}{lll}10259 & 103 & +0.18\end{array}$ $\begin{array}{lll}10024 & 60 \quad+0.12\end{array}$ $\begin{array}{llll}98 & 50 & 74 & +0.86\end{array}$ $\begin{array}{llll}98 & 22 & 37 & -0.29\end{array}$ $\begin{array}{llll}98 & 12 & 95 & -0.29\end{array}$ $\begin{array}{lll}9319 & 76 \quad+0.09\end{array}$ $\begin{array}{llll}91 & 1 & 95 & +1.24\end{array}$ $\begin{array}{llll}87 & 10 & 100 \quad+0.77\end{array}$

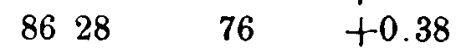
$\begin{array}{lll}8357 & 100 \quad+0.24\end{array}$ $\begin{array}{lll}8130 & 90 \quad+0.01\end{array}$ $\begin{array}{lll}77 & 19 & 21\end{array}$ $\begin{array}{llll}76 & 20 & 105 & +0.08\end{array}$ $\begin{array}{llll}7437 & 58 & +0.13\end{array}$ $\begin{array}{lll}6840 & 68 & +0.33\end{array}$ $\begin{array}{llll}6533 & 58 \quad+0.62\end{array}$ 
Hieraus resultiren nun mit Riicksicht aut die Zahl der angestellten Beobachtungen folgende Differenzen für die nebenstehenden Poldistanzen, denen ich die ent-

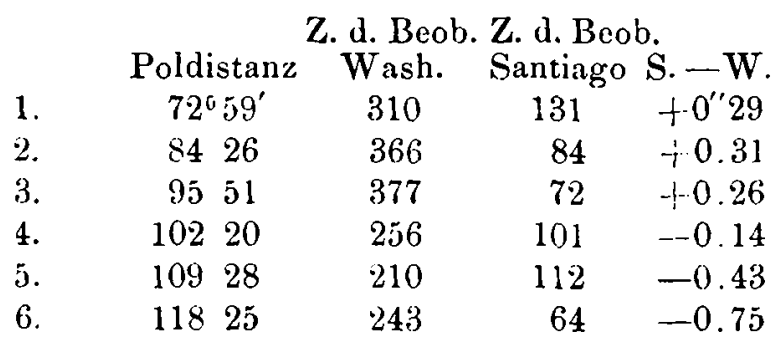

Man ersieht aus dieser \%usammenstellung, dass dic Differenzen S. - W. von den Poldistanzen abhängen, während dieses für dieselben Sterne bei dem six years Catalog nicht der Fall ist. Um den Giang der Differenzen für grössere Poldistanzen als $118^{\circ}$ weiter za verfolgen, verglich ich alle bis $-42^{\circ}$ Declination in Santiago von $1853-1860$ beobacliteten Sterne, die sich im Washingtoner Cataloge vorfinden. Von dieser Vergleichung sind nur einige wenige ansgeschlossen, bei denen entweder eine grosse eigene Bewegung wahrscheinlich ist, oder bei welchen ungewöhnliche Abweichungen Fehler in der Beobachtung oder Reduction vermuthen lassen. Die Details dieser Vergleichung werde ich in 2. Bande meiner Beobachtungen geben, dessen Druck in Kürze vollendet sein wird. Die Zahl der verglichenen, $z$ wischen den Parallelkreisen $-26^{\circ}$ und $-43^{\prime \prime}$ golegenen Sterne beträgt 288 ; auf eigene Bewegung konnte aus Mangel an soustigen genauen Beobachtungen nicht lïicksicht genommen werden; da indessen die mittleren Epochen beider Sternverzeichnisse nicht weit auscinander gehen, so lässt sich wohl annebmen, dass im Mittel aus vielen Sternen der Einfluss der E. B. auf die constanten Difterenzen klein sein wird oder gänzlich verschwindet; anderseits werden sich aber bei der Vergleichung einer grossen Anzahl von Sternen die Theilungsfehler weniger bemerklich machen, als in der obigen Zusammenstellung. Das Resultat der erwähnten Vergleichung habe ich im Folgenden zusammengefasst:

Mittel der Z. d. Beob. Z. d. Beob.

Zahl der Poldistanz Wash: Santiago S. -W. Sterne

$\begin{array}{rrrrrr}7 . & 118^{\circ} 26^{\prime} & 202 & 119 & -0^{\prime \prime} 76 & 71 \\ 8 . & 12154 & 245 & 143 & -0.93 & 102 ; \\ 9 . & 12535 & 132 & 99 & -1.04 & 64 \\ 10 . & 12939 & 123 & 98 & -1.16 & 51\end{array}$

Die Differenzen S. -W. setzen also den oben angedeuteten Gang fort. Indem ich nun den Differenzen 1-10 gleiche Gewichte beilegte und sie durch einen Ausdruck von der Form sprechenden beifüge, welche sich aus der Vergleichung mit dem six years Catalogue ergeben:

Santiago Z. d. Beob.

$\begin{array}{ccc}- \text { six years Cat. Greenw. } & \text { Sterne } \\ -0.06 & 469 & 5 \\ +014 & 305 & 4 \\ +0.28 & 332 & 5 \\ +0.12 & 312 & 4 \\ -0.58 & 322 & 5 \\ -0.14 & 108 & 2\end{array}$

$a+b \sin \delta+\cdot c \cos \delta$

darzustellen suchte, erhielt ich nach der Methode der kleinsten Quadrate folgende Formel:

$$
\text { S. - W . ‥ } 0^{\prime \prime} 101+1^{\prime \prime} 860 \sin \delta-0 " 305 \cos \delta \text {, }
$$

welche sich den beobachteten Differenzen, wie folgt, anschliesst :

$\begin{array}{ccr}\text { C. } & 0 . & \text { C. }-0 . \\ +0.65 & -0.29 & +0.36 \\ +0.28 & -0.31 & -0.03 \\ -0.08 & -0.26 & -0.34 \\ -0.29 & -0.14 & -0.15 \\ -0.51 & -0.43 & -0.08 \\ -0.75 & -0.75 & 0.00 \\ -0.75 & -0.75 & +-0.01 \\ -0.84 & -0.93 & +0.09 \\ -0.93 & -1.04 & -0.11 \\ -1.02 & --1.16 & -0.14\end{array}$

Da die Declinationen in Washiugton nur in Zehntelsecuuden gegeben sind, so erscheint diese Darstellung genügend. Man würde die obigen Differenzen gleichfalls darstellen, wollte man an die Zenithdistanzen in Santiago die Verbesserung +0 " 976 sin $\zeta$, dagegen ais die von Washington $-1^{\prime \prime} 343 \sin \zeta^{1}$ anbringen. Die Biegung des Fernrohrs in Santiago konnte wegen Maugel an geeigneten Hülfsmitteln, sowie in Folge dessen Aufstellung, die eine für dicsen Zweck nöthige sichere Placirung von Collinatoren unmöglich machte, nicht direct bestimmt werden, weshalb die in Santiago beobachteten Declinationen mit Biegung noch behaftet sind. Von der Grösse derselben wird man aber eine genäherte Kenntniss durch die in grossen Zenithdistanzen beobachteten Declinationen erlangen. Gilliss hatte während seines Aufenthalts in Santiago die Sterne $\alpha$ und $\beta$ Lyrae hauptsächlich zu dem $Z$ weck vielfach beobachtet, um die Refractionsconstante zu prüfen, da der erstere dieser Sterne nahe durch das Zenith von Washington geht und somit sowohl die Refraction als Biegung daselbst Null sind. Reducirt man nun die von Gillis beobachteten Declinationen (A Catalogue of 1963 
stars, Washington 1870) auf 1850.0 , indem man dieselben um 0"19 vergrössert, um welchen Betrag die von ihm angenommene Breite grösser ist, als die mei-

\begin{tabular}{lccrc}
\multicolumn{2}{c}{$\alpha$ Lyrae 1850.0 '/ahld. Beob. } & \multicolumn{2}{c}{$\beta$ Lyrae } & \multicolumn{2}{c}{ Zahl d. Beob. } \\
Santiago $D=51^{\prime \prime} 21^{\prime} 11^{\prime \prime} 40$ & 37 & $\mathrm{D}=56^{\prime \prime} 48^{\prime} 29^{\prime \prime} 98$ & 14 \\
Washington & 11.03 & 639 & 30.84 & 113 \\
Six years Catalog & 11.23 & 184 & 30.11 & 131
\end{tabular}

Die Vergleichung dieser Bestiumungen reigt, dass die Voraussetzung von Biegungscoefficienten, wie die obigen, nicht жulässig ist, indem für $\alpha$ Lyrae die Correction $0 " 93$ sein würde, während die Beobachtung S. -W. nur 0"37 giebt. Aus der Vergleichung des six years Catalogue, bei welchem die Biegung berücksichtigt ist und dieselben Refractionstafeln wie in Santiago und Washington gebraucht sind, folgt aber im Mittel aus beiden Sternen, dass der Einfluss der Biegung des Rohrs in Santiago verschwindend klein ist. - Auftallig bleibt bei der grossen Zahl von Beobachtungen der Unterschied zwischen Washinggton und Greenwich für $\beta$ Lyrae.

Auf alle Fälle sind die oben angegebenen Differenzen zwischen Washington und Santiago constatirt

Lucaille 8760 Epoche Z.d.Beob. AR.

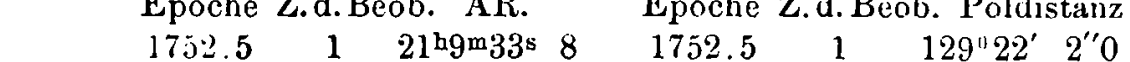

Washingtonce Kom:

Santiago

$1811 \% .7 \quad 1$

6.58

1846.7

3.79

1856.8

1.78

$1869.8 \quad 4$

$1863.6 \quad 1$

Indem ich den beiden ersten Positionen resp. dic Gewichte $1 / 10$ und $1 / 2$ beilege, bei den beiden letzteren die Gewichte der Zahl der Beobachtungen gleich setze, erhielt ich nach der Methode der kleinsten Quadrate: $\mathrm{AR} .=21^{\mathrm{b}} \mathrm{g}^{\mathrm{m}} 2^{\mathrm{s}} 83, \mathrm{D}=129^{\circ} 24^{\prime} 18^{\prime \prime} 96$. genau bestimmt, um bei Untersuchungen über eigene Bewegungen dieser Sterne in Rechnung gezogen zu werden. Für Untersuchungen dieser Art bictet aber selbstverständli.h der neue Catalog von Washington ein schätzbares Material. Ich füge noch einen der Fälle bci, in denen ein Stern bei der eben gedachten Vergleichung ausgeschlossen wurde. Es ist dieser $\mathrm{Nr}$. $9291 \mathrm{~W}$ ashingtoner Catalog oder Lacaille 8760. Dieser Stern ist auch in der Wasbingtoner Zone 94 (Merid. Transit.) beobachtet, jedoch daselbst die AR. um 1m zu gross angesetzt. Reducirt man die Angaben der verschiedenen Cataloge mit den neucren Constanten auf 1860.0 , so erhält man unter Berücksichtigung der obigen constanten Differenzen:

\begin{tabular}{crrrr} 
d. Beob. Poldistanz & \multicolumn{3}{c}{ Ausreglichen } \\
1 & $129^{\prime \prime} 22^{\prime}$ & $2^{\prime \prime} 0$ & $21^{\mathrm{hg}} 2^{\mathrm{g}} 90$ & $129^{\prime \prime} 24^{\prime} 11.0$ \\
1 & 24 & 4.7 & 2.76 & 20.66 \\
1 & 24 & 15.1 & 2.87 & 18.94 \\
4 & 24 & 30.7 & 2.81 & 18.94 \\
& m. p. in AR. $=-0^{8} 2875$ & \\
& " " " D. $=+1^{\prime \prime} 200$ &
\end{tabular}

somit eigene Bewegung im Bogen des grössten Kreises: $3^{\prime \prime} 54$.

Dresden, den 4. September 1875. C. W. Moesta.

\section{Beobachtungen kleiner Planeten auf der Sternwarte zu Kremsmünster 1875.} Meridiankreis.

Eunomia. Vergleiche B. J. B. 77.

\begin{tabular}{|c|c|c|c|c|c|c|}
\hline \multicolumn{7}{|c|}{ Mittl. Kremsm. Zeit } \\
\hline 31. Jan. & $12^{\mathbf{h}} 36^{\mathrm{m}} 15^{\mathrm{B}} 04$ & $\alpha=9^{\mathrm{h}} 19^{\mathrm{m}} 21^{\mathrm{s}} 77$ & $\mathrm{~d} \alpha=-7.57$ & $\delta=-f 8^{\prime \prime} 1^{\prime} 16^{\prime \prime} 0$ & $\mathrm{~d} \delta=+45^{\prime \prime} 2$ & $\pi=3.16$ \\
\hline 21. Fbr. & $1053 \quad 13.43$ & 85850.91 & -7.72 & +82651.9 & +49.0 & 3.04 \\
\hline 22. & 104826.88 & $\begin{array}{lll}8 & 58 & 0.13\end{array}$ & -7.76 & $+828 \quad 24.9$ & +49.0 & 3.01 \\
\hline \multicolumn{7}{|c|}{ Melpomene. B. J. B. 77.} \\
\hline 31. Jan. & 13518.20 & $9 \quad 48 \quad 29 \quad 71$ & -2.95 & +101255.0 & +2.3 & 3.40 \\
\hline 21. Fbr. & $112228.8 j$ & 92811.14 & -3.17 & +132247.3 & +3.9 & 3.11 \\
\hline $22 . \quad \gg$ & $11 \quad 1738.92$ & $\begin{array}{lll}9 & 27 & 16.97\end{array}$ & -3.17 & +133123.5 & +4.1 & 3.09 \\
\hline \multicolumn{7}{|c|}{ Sophrosyne. B. J. B. 77.} \\
\hline 3. März & $\begin{array}{lll}11 & 29 & 17.41\end{array}$ & 101426.35 & +.54 .69 & +145622.1 & $-8^{\prime} 47.5$ & 3.03 \\
\hline 5. & 111924.79 & $10 \quad 12 \quad 25.22$ & +54.75 & +145627.2 & -848.7 & 3.01 \\
\hline
\end{tabular}

